\title{
Management of common bile duct stricture caused by chronic pancreatitis with metal mesh self expandable stents
}

\author{
J Deviere, M Cremer, M Baize, J Love, B Sugai, A Vandermeeren
}

\begin{abstract}
Twenty patients with chronic pancreatitis and signs of biliary obstruction were treated by endoscopic placement of self expandable metal mesh stents, and followed up prospectively. Eleven had been treated previously with plastic endoprostheses. All had persistent cholestasis, seven patients had jaundice, and three overt cholangitis. Endoscopic stent placement was successful in all cases. No early clinical complication was seen and cholestasis, jaundice or cholangitis rapidly resolved in all patients. Mean follow up was 33 months (range 24 to 42 ) and consisted of clinical evaluation, ultrasonography, and endoscopic retrograde cholangiopancreatography (ERCP). In 18 patients, successive ERCPs and cholangioscopies have shown that the metal mesh initially embeds in the bile duct wall and is rapidly covered by a continuous tissue by three months. The stent lumen remained patent and functional throughout the follow up period except in two patients who developed epithelial hyperplasia within the stent resulting in recurrent biliary obstruction, three and six months after placement. They were treated endoscopically with standard plastic stents with one of these patients ultimately requiring surgical drainage. No patient free of clinical or radiological signs of epithelial hyperplasia after six months developed obstruction later. This new treatment could become an effective alternative to surgical biliary diversion if further controlled follow up studies confirm the initial impression that self expandable metal mesh stents offer a low morbidity alternative for longterm biliary drainage in chronic pancreatitis without the inconvenience associated with plastic stents.

(Gut 1994; 35: 122-126)
\end{abstract}

About $20 \%$ of patients with chronic pancreatitis (CP) develop common bile duct (CBD) stenosis and subsequent cholestasis or jaundice. ${ }^{1-3}$ As biliary obstruction can lead to secondary biliary cirrhosis or recurrent episodes of cholangitis, surgical treatment has been recommended for stricture associated with persistent cholestasis. ${ }^{3-5}$

Endoscopic biliary drainage using large plastic stents, is an alternative to surgery in the management of these patients, and can rapidly resolve cholestasis, jaundice, and cholangitis. Longterm results of biliary stenting, however, have been disappointing because stent clogging or migration are responsible for significant morbidity. ${ }^{2}$ For this reason, close monitoring of these patients is required, with stent replacement when necessary. Moreover, a true recalibration of the biliary stenosis after longterm plastic stenting is rarely achieved even after insertion of multiple 10 French plastic stents.

Also, surgical morbidity related to biliary drainage remains high in these alcoholic and often debilitated patients. ${ }^{3-6}$ Accordingly, it is reasonable to continue the search for alternative treatments that provide equivalent or better results with lower morbidity.

Self expandable steel braided endoprostheses were first used for the treatment of vascular and urological benign strictures, with good clinical results. ${ }^{78}$ Since then these stents have been used in bile ducts for malignant obstructive jaundice and are placed either percutaneously or endoscopically. ${ }^{9-12}$ Their use in malignant strictures provides immediate drainage, avoiding the early complications encountered with plastic stents. The longterm results are impaired, however, by tumoral overgrowth or more frequently by ingrowth through the metallic mesh into the stent, resulting in recurrent jaundice or cholangitis. ${ }^{10-12}$

As ingrowth and subsequent reocclusion seems to be tumour related, we studied this new treatment method in patients with severe CP and benign symptomatic biliary stricture.

\section{Patients and methods}

PATIENTS

Between June 1989 and January 1991, 20 patients with CP (mean age: 45 (27-61) years, four women, $16 \mathrm{men}$ ), and with signs of persistent biliary obstruction associated with CBD stricture were treated by endoscopic placement of self expandable metallic stents (Wallstent, Schneider SA, Bülach, Switzerland). All but one were alcoholics. Eleven of them had been treated previously with size 10 French plastic stents for a mean duration of 15 (2-36) months. Plastic stents were replaced by metallic stents only in case of clogging or dislodgement. Formal consent for this new type of treatment was obtained from each patient and the study conformed to our institution's guidelines concerning medical ethics.

Stenosis discovered during ERCP without persistent cholestasis, was not considered an indication for stenting. The initial indication was, in all cases, CBD stricture associated with at least persistent cholestasis (alkaline phosphatase concentrations greater than twice the normal values for more than three months and not decreasing for more than two weeks). Seven patients also had jaundice (mean bilirubin values 
$46(20-142) \mathrm{mg} / \mathrm{l})$ and three had overt cholangitis at the time of stent placement.

The mean duration of the disease from first diagnosis to the time of first biliary stent placement was 65 months (range 5-180). Four patients had cholestasis and stricture at the time of the first diagnostic procedure. Five patients had had previous surgery of the pancreas (3 lateral pancreaticojejunostomies, 2 tail pancreatectomies and 1 cyst-jejunostomy).

Figure 1: Patient no 5 : 47 year old woman with previous

pancreaticojejunostomy.

(A) appearance of the biliary stenosis after removal of a plastic stent left in place for nine months; $(B)$ the stent carrying double membrane catheter is introduced in the common bile duct and the stent is endoscopically and fluoroscopically adjusted; (C) control cholangiography after stent expansion; $(D)$ control endoscopic retrograde cholangiopancreatography 24 months later showing that the stent is covered with a

$2 \mathrm{~mm}$ thick epithelium. The lumen remains largely open.
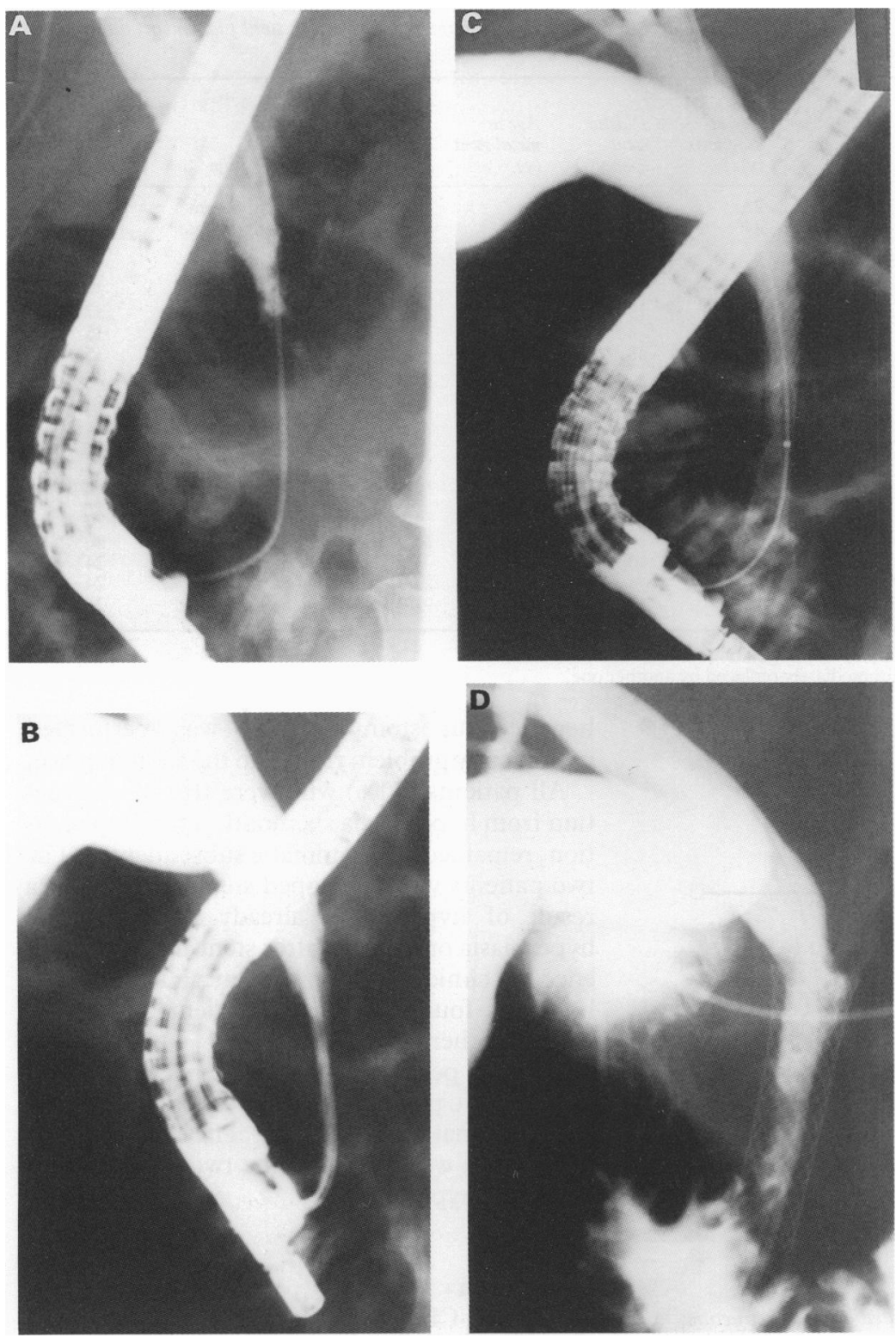
with contrast medium to $75 \mathrm{~mm} \mathrm{Hg}$ to separate the two membranes. The outer layer is then progressively withdrawn to permit stent expansion. Proximal realignment remains possible as long as the stent is not completely opened. The delivery catheter is then removed and a final opacification of the CBD is performed to assess the quality of drainage.

A $34 \mathrm{~mm}$ long stent was used in all cases. The internal diameter becomes $10 \mathrm{~mm}$ after full expansion. This length was adapted to the intrapancreatic choledocus leaving the proximal common bile duct free. The stent covered the entire stricture in each case and, as only the distal intrapancreatic portion of the CBD was stented with this unremovable material, it should not interfere with a possible future hepaticojejunostomy or choledocoduodenostomy. No dilatation was performed before or after insertion of the metal mesh stent whose complete expansion is spontaneously achieved after five days. This was seen in the first five patients treated, by serial cholangiographies through indwelling nasobiliary catheters.

\section{FOLLOW UP}

Patients were prospectively evaluated one month after stenting, by clinical examination, laboratory tests, ultrasonography, and ERCP. Thereafter, ultrasonography and laboratory tests were done every three months and ERCP every six months, during the two first years of follow up. These examinations were done on an ambulatory basis and patients were readmitted only in case of recurrent cholestasis or cholangitis. All the patients were seen in consultation and laboratory tests performed at the end of the presently reported follow up.

\section{Results}

CBD strictures corresponded to the type I of Caroli and Nora in 11 cases and type III in nine cases. The morphological appearance of the pancreatitis at ERCP consisted, in all cases, of a distal stricture of the main pancreatic duct with upstream dilatation with or without pancreatic calculi. We identify this morphology as types IV and $\mathrm{V}$ pancreatitis in our classification,,$^{13}$ these being nearly always encountered in cases of CBD stenosis in our experience. Two patients had pancreas divisum. It should be noted that 18 of these patients also had therapeutic endoscopy of the pancreas with the aim at restoring the patency of the pancreatic duct (pancreatic sphincterotomy, extracorporeal shock wave lithotripsy, with or without stent placement in the main pancreatic duct).

\section{IMMEDIATE}

ERCP was successful in all patients and in each case the stent was inserted in the CBD by the endoscopic route, during the same session; there were no sphincterotomy related complications. We encountered two technical complications: one stent was badly positioned and was left too proximally because of a technical problem during insertion. It was immediately removed with a snare and replaced with a new one. Another stent seemed, after six months, to be 
Figure 2: Alkaline phosphatase concentrations (mean $(S E M), n \leqslant 250 I U)$ after stenting. Broken line $=$ normal values.

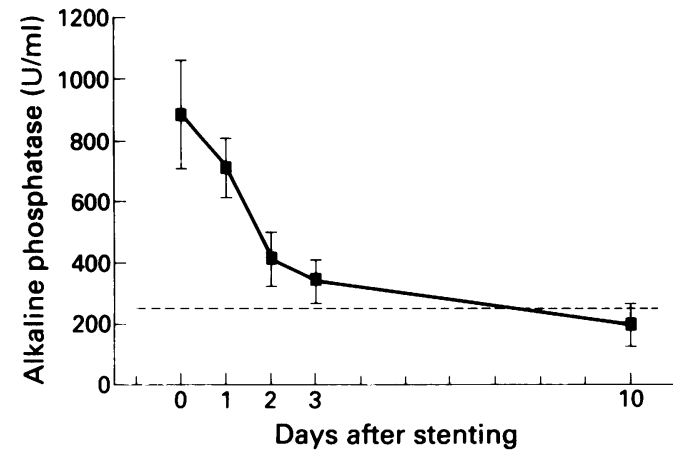

impacted in the CBD at the site of a sharp angulation. Clinically no problem was seen but we preferred to place a second stent, more proximally, overlapping the first one, to correct this angulation.

There were no problems of early clogging or dislodgement. Cholestasis, cholangitis, and jaundice resolved quickly (Fig 2) in all but one patient who continued despite adequate drainage to have slight and persistent cholestasis. Liver biopsy showed that this patient already had secondary biliary cirrhosis.
LATE

The mean follow up after common bile duct stenting is now 33 months, ranging from 24 to 42 months and is more than two years in all patients (Table, Fig 3). Eighteen patients have now been followed up for 33 (24-42) months without any biliary problems. Follow up ERCPs (in all patients) and cholangioscopies (in five patients) have shown the metal mesh to be embedded in the bile duct wall with thickened mucosa growing between the struts of the stent. At six months, the struts were usually buried by the mucosa, giving the impression of a continuous 'membrane' lining the stent. Its thickness is about $2 \mathrm{~mm}$ and the CBD lumen remains largely patent inside the stent (Fig 1). Two patients (10\%) developed epithelial hyperplasia within the stent resulting in recurrent cholestasis for one and jaundice for the other, three and six months after stent placement, respectively (Fig 4). A second self expanding stent was inserted into the first stent in each case without longterm success, as obstruction occurred again after three months in both. We then placed two plastic 10 French stents through the metallic stent. One of these patients (Table; patient 15 ) required a later

Patients' data: sex, alcoholism, previous surgery of the pancreas, age at the first diagnosis of chronic pancreatitis, duration of biliary drainage with plastic $10 \mathrm{~F}$ stents before metal stent placement, age at the time of metal mesh stent placement, date of implantation, evolution, and duration of follow up with metal stent in situ

\begin{tabular}{|c|c|c|c|c|c|c|c|c|c|}
\hline Patient & Sex & Alcoholism & $\begin{array}{l}\text { Previous } \\
\text { surgery }\end{array}$ & $\begin{array}{l}\text { Age at } \\
\text { diagnosis } \\
(y)\end{array}$ & $\begin{array}{l}\text { Plastic } \\
\text { stent } \\
\text { (months) }\end{array}$ & $\begin{array}{l}\text { Age at } \\
\text { metal stent } \\
(y)\end{array}$ & Implantation & Evolution & $\begin{array}{l}\text { Duration } \\
\text { of follow } \\
\text { up } \\
\text { (months) }\end{array}$ \\
\hline 1 & $\mathbf{M}$ & + & - & 23 & 16 & 34 & $6 / 89$ & Stent patent & 42 \\
\hline 2 & $\mathbf{F}$ & + & - & 34 & - & 36 & 9/89 & Stent patent & 39 \\
\hline 3 & M & + & + & 52 & 36 & 61 & $9 / 89$ & Stent patent & 39 \\
\hline 4 & $M$ & + & - & 27 & 18 & 34 & $9 / 89$ & Stent patent & 39 \\
\hline 5 & $\mathrm{~F}$ & + & + & 36 & 9 & 47 & $9 / 89$ & Stent patent & 39 \\
\hline 6 & M & + & - & 53 & 13 & 58 & $11 / 89$ & Stent patent & 37 \\
\hline 7 & M & + & - & 35 & 2 & 43 & $1 / 90$ & Stent patent & 35 \\
\hline 8 & $M$ & + & - & 36 & 23 & 39 & $1 / 90^{\star}$ & Stent patent & 35 \\
\hline 9 & $M$ & + & - & 29 & - & 33 & $2 / 90$ & $8 / 90$ Jaundice & 34 \\
\hline 10 & $M$ & + & - & 60 & - & 60 & $2 / 90$ & Stent patent & 34 \\
\hline 11 & M & + & + & 37 & 17 & 39 & $3 / 90$ & Stent patent & 33 \\
\hline 12 & M & + & - & 49 & - & 49 & $3 / 90$ & Stent patent & 33 \\
\hline 13 & M & + & - & 39 & 20 & 46 & $4 / 90$ & Stent patent & 32 \\
\hline 14 & M & + & - & 53 & - & 54 & $5 / 90$ & Stent patent & 31 \\
\hline 15 & $\mathbf{F}$ & - & + & 45 & - & 55 & $9 / 90$ & $12 / 90$ Cholestasis & 27 \\
\hline 16 & $\mathbf{F}$ & + & - & 27 & - & 27 & $9 / 90$ & Stent patent & 27 \\
\hline 17 & M & + & - & 38 & - & 45 & $10 / 90$ & Stent patent & 26 \\
\hline 18 & $M$ & + & + & 33 & 10 & 49 & $1 / 91$ & Stent patent & 24 \\
\hline 19 & $M$ & + & - & 43 & 4 & 46 & $1 / 91 \dagger$ & Stent patent & 24 \\
\hline 20 & $M$ & + & - & 46 & - & 47 & $1 / 91$ & Stent patent & 24 \\
\hline
\end{tabular}

^Second stent placed to correct a sharp biliary angulation

+Misplacement, stent removed and immediately replaced by another one.

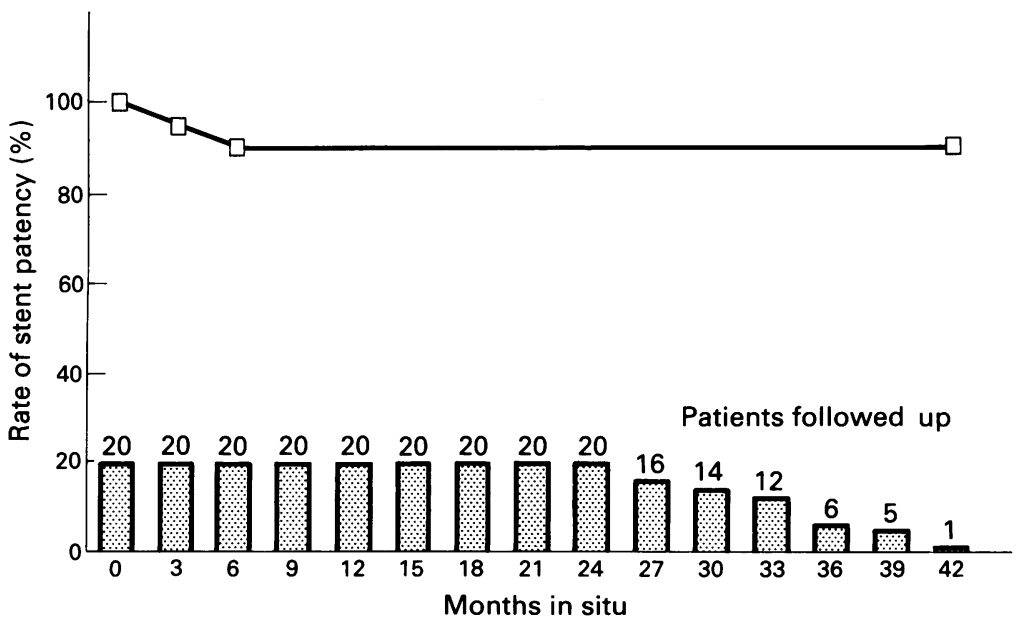

Figure 3: Probability of stent patency in chronic pancreatitis associated biliary obstruction. hepaticojejunostomy, which was performed without any problem related to the stent in place.

All patients $(90 \%)$ who were free of obstruction from hyperplasia six months after implantation, remained asymptomatic subsequently. The two patients who developed stent occlusion as a result of hyperplasia, already had abnormal hyperplasia one month after stenting. No difference in clinical or morphological features, or both were found in these two patients, compared with the others.

Biopsies performed within the stents showed hyperplastic papillary mucosa covered by biliary or intestinal epithelium. The microscopic appearance was similar in the two patients with abnormal hyperplasia.

\section{Discussion}

Persistent CBD stricture requires treatment 

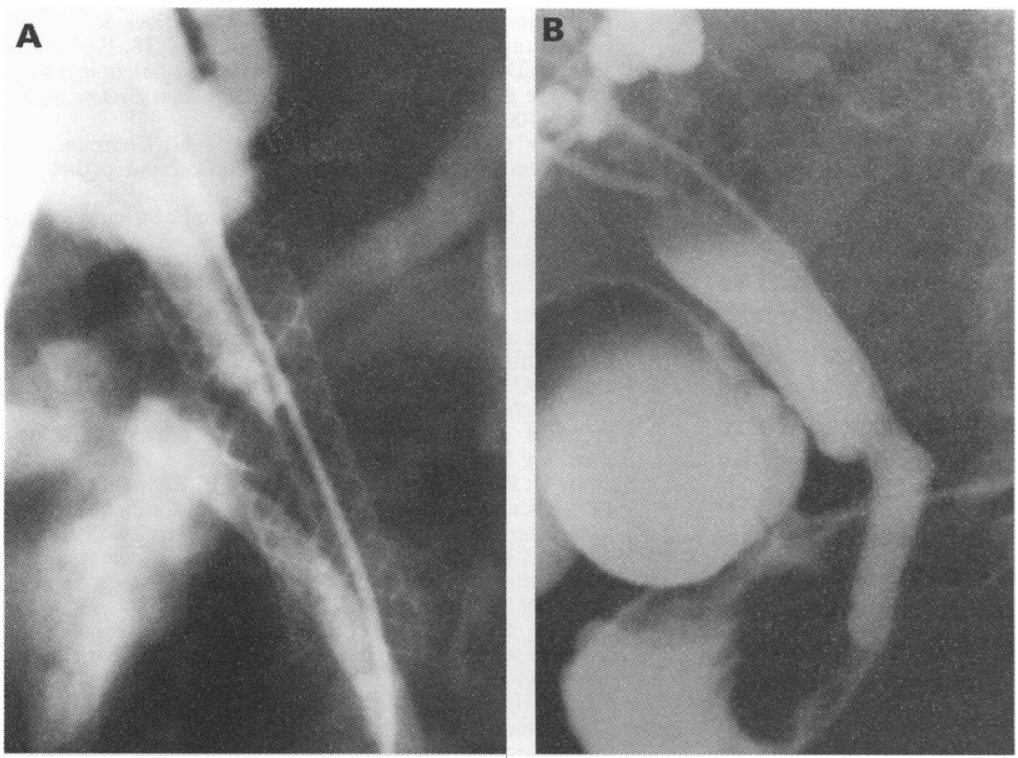

Figure 4: (A) patient no 9. Endoscopic retrograde cholangiopancreatography (ERCP) six months after metal stent placement when the patient developed jaundice because of epithelial hyperplasia into the stent. A plastic stent is in the pancreatic duct. By comparison with $(B)$, patient no 3, control ERCP 24 months after metal stent implantation.

when accompanied by a prolonged rise in serum alkaline phosphatase concentration..$^{3-5}$ This is an extremely valuable marker of significant biliary obstruction, ${ }^{6}$ which may lead to the development of hepatic damage and secondary biliary cirrhosis. In our experience, CBD stricture is seen at ERCP in $30 \%$ of patients with severe CP and is associated with persistent cholestasis, jaundice, or cholangitis in $9 \% .^{2}$ In published reports, biliary tract obstruction is the primary indication for surgery in about $10 \%$ of patients with CP. In addition, some patients having an operation aimed primarily at relieving pancreatic duct obstruction or resecting a diseased pancreas are also found to have an element of biliary tract obstruction requiring surgical drainage. ${ }^{314}$

Our initial experience of CBD drainage in CP with plastic stents was somewhat disappointing. Placement of large bore plastic biliary stents was a good initial treatment for cholangitis or jaundice when present. Longterm results, however, were unsatisfactory because of clogging or dislodgement, and required frequent replacement, which is not acceptable to these often young patients. ${ }^{2}$ Our opinion at that time was that permanent endoscopic biliary drainage in $\mathrm{CP}$ should be reserved for patients whose poor clinical condition made an operation unsuitable.

Self expandable biliary stents have been used for palliative treatment of malignant biliary strictures. All the published results available at this time show that this new material is easy to place and avoids most of the early stent related complications - that is, early clogging or dislodgement..$^{9-12}$ is The internal diameter of these stents is $10 \mathrm{~mm}$, which is three times the diameter of the largest plastic stents. To our knowledge, early clogging or dislodgement has not been reported. The initial enthusiasm concerning longterm patency of these stents in malignancies, however, has diminished because of frequent obstruction because of tumour ingrowth through the metallic struts, or tumour overgrowth. ${ }^{911}$ As obstruction in these cases is because of the tumoral process itself, we postulated that their longterm patency in benign biliary strictures was likely to be better. This was suggested by a report of the preliminary results of mesh stent implantation in postsurgical biliary stenosis. With an average follow up of eight months, no cases of reobstruction were seen in seven patients. ${ }^{14}$ These results are, however, probably too optimistic as a few cases of reocclusion by hyperplasia through the mesh have been reported with metallic stents placed either percutaneously $^{915}$ or endoscopically (C Liguory, personal communication) for postsurgical CBD strictures.

We chose to treat patients with $\mathrm{CP}$ associated biliary strictures with these devices for two reasons. The first is that we have a large number of patients with a good follow up in our institution and we are reluctant to propose surgery only for biliary diversion when there is not yet an indication for pancreatic drainage, as repeated surgery is associated with higher morbidity. The second is that the CBD stricture is always distal allowing for the use of comparatively short (34 mm) mesh stents.

Indeed, as these stents are not removable and because we are treating patients with benign disease, we did not want to compromise a potential surgical biliary diversion. As the stent is left in the distal part of the CBD, a future hepaticojejunostomy or choledochoduodenostomy still remains possible as was shown in one of our patients.

The potential for malignant transformation at the site of implantation as a result of chronic irritation from the metal remains a theoretical problem worthy of consideration. Clearly, this study cannot provide a definitive answer. Surgeons, however, have used metallic implants such as clips and automatic sutures for a long time without any such complication reported. ${ }^{16}$ It seems therefore that implanted metal despite a potential to induce fibrosis or inflammation, has not been responsible for malignancy. Also reassuring are animal studies in which these stents have shown excellent biological tolerance and no dysplastic changes have been noted. ${ }^{17}$

Ninety per cent of stents remain patent at three years and when abnormal hyperplasia occurs, resulting in stent occlusion, it occurs during the first six months. Two of our 20 patients required further biliary drainage. Epithelial hyperplasia is a constant finding in the presence of these stents, probably resulting from mechanical irritation. The reason why it becomes more pronounced in some patients remains unanswered. In the two cases of reobstruction, the pattern mimicked the previous stricture, suggesting that the radial force exerted by the mesh on the wall may have led to deeper embedding of the mesh where the stricture was tight or may have provoked more important hyperplasia, or both. This may partially explain the better results seen here compared with those obtained with metal stents in postsurgical strictures. ${ }^{15}$ In these cases, the stricture is short and tight and the radial force exerted by the individual wires at the level of the stricture is therefore higher.

The lower end of the stent must be level with the papilla to avoid the presence of metallic mesh within the duodenum and must extend over the entire stricture. In the event of misplacement, as we saw in one patient, immediate removal is 
possible. Where there is sharp angulation of the CBD, the upper part of the stent should be $1 \mathrm{~cm}$ above or $1 \mathrm{~cm}$ below the angulation, but not at the point of angulation itself, to avoid impaction of the metallic mesh in the CBD wall and possibly further impaired drainage.

Within a month of placement, the metallic mesh embeds into the bile duct wall and, after three months, the struts are buried by the mucosa, giving the impression of a continuous membrane covering the inner stent. This finding is comparable with animal studies, ${ }^{17}$ where implantation of metal stents is followed by mucosal hyperplasia through the space between the struts, which finally are buried by the mucosa. This finding is suggestive of destruction of the original mucosal layer during expansion with subsequent healing and proliferation. 'Clogging' of these stents by material comparable with the one seen in plastic stents has not been seen, probably because of both a larger diameter of the lumen and the nature of the stent's inner lining after re-epithelialisation.

Longer follow up and possibly controlled clinical trials are needed to determine if metal stents have the potential to become the first choice treatment for persistent, symptomatic CP associated biliary stricture. This study suggests, however, that there may be a satisfactory low morbidity alternative to biliary surgery providing efficient prolonged biliary drainage, without the usual complications and discomfort associated with plastic stents.

The authors thank G Ghattas, MD, and C Matthys for their help in proof reading and typing the manuscript.
1 Scott J, Summerfield JA, Elias E, Dick R, Sherlock S. Chronic pancreatitis: a cause of cholestasis. Gut 1977; 18: 196-201.

2 Deviere J, Devaere S, Baize M, Cremer M. Endoscopic biliary drainage in chronic pancreatitis. Gastrointest Endosc 1990; 36: $96-100$.

3 Littenberg G Afroudakis A, Kaplowitz N. Common bile duct stenosis from pancreatitis: a clinical and pathologic duct stenosis from pancreatitis: a clinica

4 Stabile BE, Calabria R, Wilson SE, Passaro E. Stricture of the common bile duct from chronic pancreatitis. Surg Gynecol common bile duct from

5 Warshaw AL, Shapiro RH, Ferrucci JJ, Galdabani JJ. Persistent obstructive jaundice, cholangitis and biliary cirrhosis due to common bile duct stenosis in chronic pancreatitis. Gastroenterology 1976; 70: 562-7.

6 Stahl TJ, O'Connor Allen M, Ansel HJ, Vennes JA. Partial biliary obstruction caused by chronic pancreatitis. An approval of indications for surgical biliary drainage. Ann Surg 1988; 207: 26-32.

7 Sigwart U, Puel J, Mirkovitch V, Joffre F, Kappenberger L. Intravascular stents to prevent reclusion and restenosis after Intravascular stents to prevent reclusion and restenosis after

8 Milroy E, Chapple C, El Din A, Wallsten H. A new stent for the treatment of urethral strictures. Preliminary report. the treatment of urethral
$B r \mathcal{F}$ Urol 1989; 63: $392-6$.

9 Gillams A, Dick R, Dooley J, Wallsten H. Self expandable stainless steel braided endoprosthesis for biliary strictures. Radiology 1990; 174: 137-40.

10 Neuhaus H, Hagenmuller F, Griebel M, Classen M. Self expanding metal stents versus conventional plastic endoprostheses for malignant biliary obstruction [Abstract] Gastrointest Endosc 1991; 37: 253

11 Cremer M, Deviere J, Sugai B, Baize M. Expandable biliary metal stents for malignancies: endoscopic insertion and diathermic cleaning for tumor ingrowth. Gastrointest Endosc 1990; 36: 451-7.

12 Huibregtse K, Cheng J, Coene PPL, Tytgat GNJ. Endoscopic placement of expandable metal stents for biliary strictures. placement of expandable metal stents for biliary strictures. Andoscopy $1989 ; 21: 280-5$.

13 Delhaye $M$, Vandermeeren A, Baize M, Cremer M. Extra corporeal shock wave lithotripsy of pancreatic calculi. Gastroenterology 1992; 102: 610-20.

14 Foerster EC, Hoepffner N, Domschke W. Bridging of benign choledocal stenoses by endoscopic retrograde implantation of mesh stents. Endoscopy 1991; 23: 133-5.

15 Maccioni F, Rossi M, Salvatori FP, Ricci P, Bezzi M, Rossi P. Metallic stents in benign biliary strictures: three year followup. Cardiovasc Intervent Radiol 1992; 15: 360-8.

16 Fekete F, Gayet B, Place S, Biagrin J. Four hundred oesophageal anastomoses with a stapler. Int Surg 1989; 74: 0esoph.

17 Alvarado R, Palmaz JC, Garcia OJ, Tio FO, Rees CR. Evaluation of polymer coated balloon expandable stents in bile ducts. Radiology 1989; 170: 975-8. 